\title{
Two New Reformulation Convexification Based Hierarchies for 0-1 MIPs
}

\author{
Hacene Ouzia \\ Sorbonne Universités, UPMC Univ Paris 06, LIP6 UMR 7606, 4 Place Jussieu, 75005 Paris, France \\ Correspondence should be addressed to Hacene Ouzia; hacene.ouzia@upmc.fr
}

Received 28 July 2015; Accepted 5 October 2015

Academic Editor: Ching-Jong Liao

Copyright (C) 2015 Hacene Ouzia. This is an open access article distributed under the Creative Commons Attribution License, which permits unrestricted use, distribution, and reproduction in any medium, provided the original work is properly cited.

First, we introduce two new reformulation convexification based hierarchies called RTC and RSC for which the rank $d$ continuous relaxations are denoted by $\widehat{P}_{\mathrm{RTC}}^{d}$ and $\widehat{P}_{\mathrm{RSC}}^{d}$, respectively. These two hierarchies are obtained using two different convexification schemes: term convexification in the case of the RTC hierarchy and standard convexification in the case of the RSC hierarchy. Secondly, we compare the strength of these two hierarchies. We will prove that (i) the hierarchy RTC is equivalent to the RLT hierarchy of Sherali-Adams, (ii) the hierarchy RTC dominates the hierarchy RSC, and (iii) the hierarchy RSC is dominated by the Lift-and-Project hierarchy. Thirdly, for every rank $d$, we will prove that $\operatorname{conv}\left(\mathscr{T}^{d} \cap \mathscr{E}_{t}^{d}\right) \subseteq \widehat{P}_{\mathrm{RTC}}^{d} \subseteq \mathscr{T}^{d}$ and $\operatorname{conv}\left(\mathcal{S}^{d} \cap \mathscr{E}_{s}^{d}\right) \subseteq \widehat{P}_{\mathrm{RSC}}^{d} \subseteq \mathcal{S}^{d}$ where the sets $\mathscr{T}^{d}$ and $\mathcal{S}^{d}$ are convex, while $\mathscr{E}_{t}^{d}$ and $\mathscr{E}_{s}^{d}$ are two nonconvex sets with empty interior (all these sets depend on the convexification step). The first inclusions allow, in some cases, an explicit characterization (in the space of the original variables) of the RLT relaxations. Finally, we will discuss weak version of both RTC and RSC hierarchies and we will emphasize some connections between them.

\section{Introduction}

Let $n$ and $m$ be two integers. Let $E$ and $N$ be the two sets $\{1, \ldots, n\}$ and $\{1, \ldots, n+m\}$, respectively. Let $X$ be a subset of $\mathbb{R}_{+}^{n+m}$ representing the set of feasible solutions of a mixed integer linear program. The integer $n$ indicates the number of binary variables. We will assume that the set $X$ is bounded and has the following nonlinear description:

$$
\begin{aligned}
\sum_{j \in N} a^{j} x_{j} & \leq b, \\
x_{j} & \leq 1, \quad j \in N, \\
-x_{j} & \leq 0, \quad j \in N, \\
x_{j} & \in\{0,1\}, \quad j \in E .
\end{aligned}
$$

In descriptions (1)-(4) above the set $E$ contains the indices of the $n$ binary variables describing $X$; for each index $j$ belonging to $N$ the vectors $a^{j}$ and $b$ belong to $\mathbb{R}^{\rho}$, where $\rho$ is a positive integer indicating the number of constraints in (1). The $i$ th component of the two vectors $a^{j}$ and $b$ will be denoted by $a_{i j}$ and $b_{i}$, respectively. Finally, $\bar{X}$ defined by the constraints (1), (2), and (3) will denote the continuous (or linear) relaxation of the mixed integer set $X$.

In the sequel, two linear descriptions are said to be equivalent if they define the same polyhedron. A linear description $D_{1}$ dominates another linear description $D_{2}$ if the polyhedron defined by $D_{1}$ is included in the polyhedron defined by $D_{2}$.

Optimizing even a linear function over the mixed integer set $X$ is an NP-hard problem in general (see [1-4]). A way of building strengthened linear relaxations is to use an approach combining reformulation, linearization, and projection such as those proposed in [5-10]. In such approach, one first reformulates the constraints defining the set of feasible solutions by introducing nonlinearities. Then, the resulting nonlinear system is linearized and projected back onto the original space.

Two important properties characterize the reformulationlinearization approach (also known as Lift-and-Project methods (do not confuse this with the Lift-and-Project hierarchy introduced by Balas et al., see [5])). First, the approach leads to a whole hierarchy (see $[10,11])$ of relaxations which lie 
between the continuous relaxation $\bar{X}$ and the convex hull of the mixed integer set $X$. And for a given hierarchy, a relaxation of higher rank (see $[10,11]$ ) is always stronger than a relaxation of lower rank. Secondly, optimizing a linear function over any relaxation of the hierarchy can be done in a polynomial time.

Many hierarchies were introduced. To mention a few, L\&P hierarchy (Balas et al., see [5]), LS hierarchy (Lovász and Schrijver, see [7]), RLT hierarchy (Sherali and Admas, see $[9,10]$ ), LAS hierarchy (Lasserre, see [6]), BZ hierarchy (Bienstock and Zuckerberg, see [12]), and DRL * hierarchy (Minoux and Ouzia, see $[8,13]$ ). For more details, a set-theoretical interpretation of the reformulationlinearization approaches has been proposed in [14] and a theoretical comparative study between the RLT, LS, and LAS relaxations can be found in [15].

The convexification technique is also widely used to solve nonlinear and nonconvex optimization problems (see [16] and the references therein). Roughly speaking, this technique consists in approximating a nonconvex optimization problem by a convex problem (or a family of convex problems). This can be done by approximating the nonconvex objective function by a convex function and/or by approximating the nonconvex set of feasible solutions by a convex one (see [17$24]$ and the references therein). In this paper, new hierarchies of continuous relaxations using a reformulation, convexification, and linearization approach will be defined. Theses hierarchies are obtained using two different convexification schemes.

The paper is organized as follows. In the second section, first, we will recall the definition of a reformulationlinearization hierarchies and then give the definition of the Sherali-Adams hierarchy. In the third section, we will define two reformulation-convexification hierarchies: RTC and RSC hierarchies. We will study the main properties of these new hierarchies. In the fourth section, we will study the connections between RTC, RSC, and L\&P hierarchies. In the fifth section, we will introduce a weak version of the hierarchies RTC and RSC and emphasize some connections between them. In the last section we make some concluding remarks.

\section{The Reformulation-Linearization Hierarchies}

First, we will introduce the general concept of reformulationlinearization hierarchies. Then, we recall the definition of the well-known Sherali-Adams hierarchy (for more details see [9, 10, 25]).

Let $p$ be a positive integer. For a finite nonempty set $S$ let $S^{[p]}$ be the set of all subsets of $S$ with cardinality $p$, whereas $S^{(p)}$ is the set of all subset of $S$ with cardinality at most $p$. Sometimes we will need to indicate the cardinality of the sets under consideration, so we will use the notation $H^{p}$ to indicate (do not confuse this with the Cartesian product of sets) that the set $H$ has cardinality $p$.

Let $J^{d}$ be a set of $d$ elements belonging to the set of binary indices $E$ and let $J$ be a subset from $J^{d}$. We call $d$-factor associated with the sets $J$ and $J^{d}$, denoted by $F_{d}\left(J, J^{d} \backslash J\right)$, the degree $d$ polynomial defined as follows:

$$
F_{d}\left(J, J^{d} \backslash J\right)=\prod_{j \in J} x_{j} \prod_{j \in J^{d} \backslash J}\left(1-x_{j}\right),
$$

with the convention that $\prod_{j \in \emptyset} y_{j}=1$.

Example 1. In the case where $E=\{1,2,3\}$ and $d=2$ we have the following 12 nontrivial 2-factors: $x_{1} x_{2}, x_{1} x_{3}, x_{2} x_{3}$, (1$\left.x_{1}\right) x_{2},\left(1-x_{1}\right) x_{3},\left(1-x_{2}\right) x_{1},\left(1-x_{2}\right) x_{3},\left(1-x_{3}\right) x_{1},\left(1-x_{3}\right) x_{2}$, $\left(1-x_{1}\right)\left(1-x_{2}\right),\left(1-x_{1}\right)\left(1-x_{3}\right)$, and $\left(1-x_{2}\right)\left(1-x_{3}\right)$.

A rank $d$ reformulation-linearization relaxation (of the mixed integer set $X$ described by (1)-(4)) is defined in three steps. First, the problem is reformulated as a $0-1$ polynomial (semialgebraic (a $n$-dimensional semialgebraic set is a solution set of a finite system of polynomial equalities and inequalities; for more details see $[26,27]))$ mixed integer system by multiplying constraints (1)-(3) with all possible $d$-factors (that is multiplying by $F_{d}\left(J, J^{d} \backslash J\right.$ ) for all subsets $J^{d}$ of $E$ and all $J \subseteq J^{d}$ ). Then, the nonlinear terms are linearized by replacing them with new variables giving rise to a higher dimensional linear system. The third step consists in projecting back the resulting polyhedron onto the original $x$-space. As observed in [8] the linearization step can be performed in various ways, leading to various hierarchies of relaxations.

The solution set in $\mathbb{R}^{n+m}$ associated with the nonlinear (semialgebraic) description resulting from the reformulation step will be denoted by $R^{d}$ and it is defined as follows:

$$
R^{d}=\bigcap_{J^{d} \in E^{[d]}} R^{d}\left(J^{d}\right)
$$

where, for each subset $J^{d}$ of $E, R^{d}\left(J^{d}\right)$ is the solution set defined by the following nonlinear system:

$$
\begin{gathered}
\sum_{j \in N} a^{j} x_{j} F_{d}\left(J, J^{d} \backslash J\right) \leq b F_{d}\left(J, J^{d} \backslash J\right), \quad J \subseteq J^{d}, \\
x_{j} F_{d}\left(J, J^{d} \backslash J\right) \leq F_{d}\left(J, J^{d} \backslash J\right), \\
\quad j \in N, J \subseteq J^{d}, \\
-x_{j} F_{d}\left(J, J^{d} \backslash J\right) \leq 0, \quad j \in N, J \subseteq J^{d}, \\
-F_{d}\left(J, J^{d} \backslash J\right) \leq 0, \quad J \subseteq J^{d} .
\end{gathered}
$$

Starting from this semialgebraic reformulation, various linear relaxations can be constructed depending on the type of linearization considered (for more details see $[8,13]$ ).

2.1. The Sherali-Adams Hierarchy. The description of the rank $d$ Sherali-Adams relaxation for the mixed integer set $X$ defined by (1)-(4), denoted by $\widehat{P}_{\mathrm{RLT}}^{d}$, is a reformulationlinearization relaxation of rank $d$ where the nonlinear terms 
appearing in (7) are linearized by introducing a new set of variables $w_{J}$ and $w_{J}^{k}$ defined by

$$
\begin{aligned}
& w_{J}=\prod_{j \in J} x_{j} \quad \forall J \subseteq E \text { with }|J| \leq \min \{d+1, n\}, \\
& w_{J}^{k}=x_{k} \prod_{j \in J} x_{j}
\end{aligned}
$$

$\forall k \in N \backslash E, J \subseteq E$ with $|J| \leq \min \{d, n\}$,

where it is assumed that $w_{\emptyset}=1$ and $w_{\emptyset}^{k}=x_{k}$ for every index $k$ (belonging to $N \backslash E$ ) of a continuous variable.

Example 2. In the case where $E=\{1,2,3\}$ and $N=E \cup\{4\}$ we have the following linearization:

$$
\begin{gathered}
w_{\{1,2\}}=x_{1} x_{2}, \\
w_{\{1,3\}}=x_{1} x_{3}, \\
w_{\{2,3\}}=x_{2} x_{3}, \\
w_{\{1\}}^{4}=x_{1} x_{4}, \\
w_{\{2\}}^{4}=x_{2} x_{4}, \\
w_{\{3\}}^{4}=x_{3} x_{4} .
\end{gathered}
$$

The resulting higher dimensional linear description will be denoted by $P_{\mathrm{RLT}}^{d}$ and it is defined as follows:

$$
P_{\mathrm{RLT}}^{d}=\bigcap_{J^{d} \in E^{[d]}} P_{\mathrm{RLT}}^{d}\left(J^{d}\right),
$$

where, for each cardinality $d$ subset $J^{d}$ of $E$, the linear description of the polyhedron $P_{\mathrm{RLT}}^{d}\left(J^{d}\right)$ is

$$
\begin{aligned}
\sum_{j \in N} a^{j} W_{j}^{J, J^{d}}-b W_{0}^{J, J^{d}} & \leq 0, \quad J \subseteq J^{d}, \\
W_{j}^{J, J^{d}}-W_{0}^{J, J^{d}} & \leq 0, \quad j \in N, \quad J \subseteq J^{d}, \\
W_{j}^{J, J^{d}} & \geq 0, \quad j \in N, J \subseteq J^{d}, \\
W_{0}^{J, J^{d}} & \geq 0, \quad J \subseteq J^{d},
\end{aligned}
$$

and where, for every index $j$ belonging to $N \backslash E, W_{j}^{J, J^{d}}$ and $W_{0}^{J J^{d}}$ denote the linearized forms of the polynomials $x_{j} F_{d}\left(J, J^{d} \backslash J\right)$ and $F_{d}\left(J, J^{d} \backslash J\right)$, respectively; these are related to the $w_{J}$ and $w_{J}^{k}$ variables as follows:

$$
\begin{aligned}
& W_{j}^{J, J^{d}}=\sum_{J \subseteq H \subseteq J^{d}}(-1)^{|H \backslash J|} w_{H \cup\{j\}}, \quad J^{d}, J \subseteq J^{d}, j \in E, \\
& W_{j}^{J, J^{d}}=\sum_{J \subseteq H \subseteq J^{d}}(-1)^{|H \backslash J|} w_{H}^{j}, \quad J^{d}, J \subseteq J^{d}, j \in N \backslash E, \\
& W_{0}^{J, J^{d}}=\sum_{J \subseteq H \subseteq J^{d}}(-1)^{|H \backslash J|} w_{H}, \quad J^{d}, J \subseteq J^{d} .
\end{aligned}
$$

The above relations (12) are easily obtained by expanding the products involved in the definition of the $d$-factors.

After linearizing the nonlinear terms in (7) using the $w$ variables defined in (8) above, the $P_{\mathrm{RLT}}^{d}$ description turns out to involve a number of variables and constraints exponential in $d$. The number of variables needed to linearize the nonlinear system (7) is $\sum_{k=1}^{\min \{d+1, n\}}\left(\begin{array}{l}n \\ k\end{array}\right)+m \sum_{k=0}^{d}\left(\begin{array}{l}n \\ k\end{array}\right)$ (notice that the variable $w_{\emptyset}$ is not counted here since $\left.w_{\emptyset}=1\right)$. Also, it is seen that the number of constraints is $O\left(\left(\begin{array}{l}n \\ d\end{array}\right) 2^{d}(n+m)\right)$.

The rank $d$ Sherali-Adams relaxation $\widehat{P}_{\mathrm{RLT}}^{d}$ is obtained by projecting the polyhedron $P_{\mathrm{RLT}}^{d}$ onto the subspace $\mathbb{R}^{n+m}$ of the $x$ variables.

\section{Two New Reformulation-Convexification Hierarchies}

We will consider two new reformulation-convexification hierarchies. The first one is called reformulation-termconvexification (RTC) hierarchy. It is obtained by convexifying the monomials (also called terms) resulting from the reformulation step. The second hierarchy is called reformulationstandard-convexification (RSC). It is obtained by convexifying the nonlinear factors (a linear combination of monomial products) resulting from the reformulation step.

3.1. Reformulation-Term-Convexification Hierarchy. A rank $d$ relaxation of the RTC hierarchy is obtained by applying local convexification to each constraint of the nonlinear system defining $R^{d}$ as follows. For every subset $J$ from $E$ and every subset $H$ from $N \backslash E$ with at most one element, let $l$ be the following operator:

$$
\begin{aligned}
& l\left(\alpha \prod_{k \in H} x_{k} \prod_{j \in J} x_{j}\right) \\
& \quad= \begin{cases}\alpha\left(\sum_{j \in J \cup H} x_{j}-|J \cup H|+1\right)^{+} & \text {if } \alpha>0, \\
\alpha \min \left\{x_{j}: j \in J \cup H\right\} & \text { if } \alpha \leq 0,\end{cases}
\end{aligned}
$$

with the convention that $\prod_{k \in \emptyset} x_{k}=1$ and, for any real $r,(r)^{+}$ is equal to $\max \{0, r\}$.

The convexification scheme (13) assumes that the constraint to which it is applied is of the form $\leq$.

Let $\mathscr{T}^{d}$ be the following convex set:

$$
\mathscr{T}^{d}=\bigcap_{J^{d} \in E^{[d]}} \mathscr{T}^{d}\left(J^{d}\right),
$$

where, for each cardinality $d$ subset $J^{d}$ from $E$, the convex set $\mathscr{T}^{d}\left(J^{d}\right)$ corresponds to the solution set $R^{d}\left(J^{d}\right)$ defined by the nonlinear system deduced from (7) by convexification 
using scheme (13). The convex nonlinear description of the set $\mathscr{T}^{d}\left(J^{d}\right)$, for a given $d$, reads

$$
\begin{gathered}
\sum_{j \in N} L_{j}^{J, J^{d}}\left[a_{i j}\right]+L_{0}^{J J J^{d}}\left[-b_{i}\right] \leq 0, \quad J \subseteq J^{d}, i=1, \ldots, \rho, \\
L_{j}^{J J^{d}}[1]+L_{0}^{J, J^{d}}[-1] \leq 0, \quad j \in N, J \subseteq J^{d}, \\
L_{j}^{J, J^{d}}[1] \geq 0, \quad j \in N, J \subseteq J^{d}, \\
L_{0}^{J, J^{d}}[1] \geq 0, \quad J \subseteq J^{d},
\end{gathered}
$$

where, for every index $j$ belonging to $N$ and any scalar $\sigma, L_{j}^{J J^{d}}[\sigma]$ and $L_{0}^{J, J^{d}}[\sigma]$ denote the convexified forms of the polynomials $\sigma x_{j} F_{d}\left(J, J^{d} \backslash J\right)$ and $\sigma F_{d}\left(J, J^{d} \backslash J\right)$, respectively; these are defined using the $l$ operator as follows:

$$
\begin{gathered}
L_{j}^{J, J^{d}}[\sigma]=\sum_{J \subseteq H \subseteq J^{d}} l\left(\sigma \times(-1)^{|H \backslash J|} \prod_{k \in H \cup\{j\}} x_{k}\right), \\
J^{d}, J \subseteq J^{d}, j \in E, \\
L_{j}^{J, J^{d}}[\sigma]=\sum_{J \subseteq H \subseteq J^{d}} l\left(\sigma \times(-1)^{|H \backslash J|} x_{j} \prod_{k \in H} x_{k}\right), \\
J^{d}, J \subseteq J^{d}, j \in N \backslash E, \\
L_{0}^{J J^{d}}[\sigma]=\sum_{J \subseteq H \subseteq J^{d}} l\left(\sigma \times(-1)^{|H \backslash J|} \prod_{k \in H} x_{k}\right), \\
J^{d}, J \subseteq J^{d} .
\end{gathered}
$$

Since the set $\mathscr{T}^{d}$ is the intersection of convex sets (by construction), then we have the following result.

Theorem 3. For every integer $d$ belonging to $\{1, \ldots, n\}$, the set $\mathscr{T}^{d}$ as defined by (15) is a nonlinear convex relaxation of the mixed integer set $X$.

For every integer $d$, let $\mathscr{E}_{t}^{d}$ be the set (the lower-script $t$ is used to recall that our set is related to the term convexification scheme):

$$
\mathscr{E}_{t}^{d}=\bigcap_{H \in E^{(d)}, K \in(N \backslash H)^{(1)}} \mathscr{E}_{t}(H \cup K),
$$

where, for subsets $H \in E^{(d)}$ and $K \in(N \backslash H)^{(1)}$,

$$
\begin{aligned}
& \mathscr{E}_{t}(H \cup K)=\{x \\
& \in[0,1]^{n+m}:\left(\sum_{j \in H \cup K} x_{j}-|H \cup K|+1\right)^{+} \\
& \left.\quad=\min \left\{x_{j}: j \in H \cup K\right\}\right\} .
\end{aligned}
$$

Since every binary vector from $\{0,1\}^{n+m}$ belongs to $\mathscr{E}_{t}^{d}$ then we deduce that $\operatorname{conv}\left(\mathscr{E}_{t}^{d}\right)$ coincides with the hypercube $[0,1]^{n+m}$.

Let $P_{\mathrm{RTC}}^{d}$ be the extended linear description obtained from the set $\mathscr{T}^{d} \cap \mathscr{E}_{t}^{d}$ using the following steps. Let $t$ and $v$ be two sets of additional variables such that for every $d$-element set $J^{d}$, for every subset $J$ from $J^{d}$ and for every subset $H$ from $N \backslash J^{d}$ with at most one element. First, in (15), the variable $t_{J}^{H}$ will replace the nonlinear term:

$$
\min \left\{x_{j}: j \in J \cup H\right\},
$$

and the variable $v_{J}^{H}$ will replace

$$
\left(\sum_{j \in J \cup H} x_{j}-|J \cup H|+1\right)^{+} .
$$

Then, we impose the equality constraint:

$$
t_{J}^{H}=v_{J}^{H} .
$$

We will use the notation $t_{J}$ or $t_{J}^{k}$ instead of $t_{J}^{H}$ when $H$ is empty or when $H$ coincides with the singleton $\{k\}$, respectively.

Thus, we have

$$
P_{\mathrm{RTC}}^{d}=\bigcap_{J^{d} \in E^{[d]}} P_{\mathrm{RTC}}^{d}\left(J^{d}\right),
$$

where, for each subset $J^{d}$ of $E$, the polyhedron $P_{\mathrm{RTC}}^{d}\left(J^{d}\right)$ reads

$$
\begin{aligned}
& \sum_{j \in N} a^{j} T_{j}^{J, J^{d}}-b T_{0}^{J J^{d}} \leq 0, J \subseteq J^{d}, \\
& T_{j}^{J J^{d}}-T_{0}^{J J J^{d}} \leq 0, j \in N, J \subseteq J^{d}, \\
&-T_{j}^{J J J^{d}} \leq 0, \quad j \in N, \quad J \subseteq J^{d}, \\
&-T_{0}^{J J J^{d}} \leq 0, \quad J \subseteq J^{d}, \\
& \min \left\{x_{j}: j \in J\right\} \geq t_{J}, \quad J \subseteq J^{d}, \\
& \sum_{j \in J} x_{j}-|J|+1 \leq t_{J}, \quad J \subseteq J^{d}, \\
& \min \left\{x_{j}: j \in J \cup\{k\}\right\} \geq t_{J}^{k}, \quad J \subseteq J^{d}, k \in N \backslash E, \\
& x_{k}+\sum_{j \in J} x_{j}-|J| \leq t_{J}^{k}, \quad J \subseteq J^{d}, k \in N \backslash E, \\
& t_{J}, t_{J}^{k} \geq 0, \quad J \subseteq J^{d}, k \in N \backslash E,
\end{aligned}
$$

and where, for every index $j$ belonging to $N, T_{j}^{J, J^{d}}$ and $T_{0}^{J, J^{d}}$ denote the linearized forms of the convexified form of the 
polynomials $x_{j} F_{d}\left(J, J^{d} \backslash J\right)$ and $F_{d}\left(J, J^{d} \backslash J\right)$, respectively; these are related to the $t_{J}$ and $t_{J}^{k}$ variables as follows:

$$
\begin{aligned}
& T_{j}^{J J^{d}}=\sum_{J \subseteq H \subseteq J^{d}}(-1)^{|H \backslash J|} t_{H \cup\{j\}}, \quad J^{d}, J \subseteq J^{d}, j \in E, \\
& T_{j}^{J, J^{d}}=\sum_{J \subseteq H \subseteq J^{d}}(-1)^{|H \backslash J|} t_{H}^{j}, \quad J^{d}, J \subseteq J^{d}, j \in N \backslash E, \\
& T_{0}^{J, J^{d}}=\sum_{J \subseteq H \subseteq J^{d}}(-1)^{|H \backslash J|} t_{H}, \quad J^{d}, J \subseteq J^{d} .
\end{aligned}
$$

The linear description (23)-(31) and (32) are stated using only the variables $t_{J}^{H}$. This is possible according to (21). As discussed in Section 5, discarding constraints (21) in the definition of the RTC hierarchy will lead to a weaker hierarchy.

For every integer $d$ belonging to $\{1, \ldots, n\}$, let $\widehat{P}_{\text {RTC }}^{d}$ be the projection onto the $x$-space of the extended linear description $P_{\mathrm{RTC}}^{d}$. The polyhedron $\widehat{P}_{\mathrm{RTC}}^{d}$ will be called rank- $d$ reformulation-term-convexification relaxation of $X$.

In the next theorem we will prove that the hierarchy RTC is equivalent to the hierarchy RLT.

Theorem 4. For every integer d belonging to $\{1, \ldots, n\}$, the two linear relaxations $\widehat{P}_{\mathrm{RLT}}^{d}$ and $\widehat{P}_{\mathrm{RTC}}^{d}$ are equivalent.

Proof. We will proceed by showing that the two extended linear descriptions $P_{\mathrm{RLT}}^{d}$ and $P_{\mathrm{RTC}}^{d}$ are the same up to variable renaming. Let $d$ be an integer belonging to $\{1, \ldots, n\}$. As shown in [8], for every set $J^{d}$ belonging to $E^{[d]}$, the constraints

$$
\begin{gathered}
\min \left\{x_{j}: j \in J\right\} \geq w_{J}, \quad J \subseteq J^{d}, \\
\sum_{j \in J} x_{j}-|J|+1 \leq w_{J}, \quad J \subseteq J^{d},
\end{gathered}
$$

are implicit in the linear description of $P_{\mathrm{RLT}}^{d}$. We claim that the constraints

$$
\begin{array}{r}
\min \left\{x_{j}: j \in J \cup\{k\}\right\} \geq w_{J}^{k}, \quad J \subseteq J^{d}, k \in N \backslash E, \\
x_{k}+\sum_{j \in J} x_{j}-|J| \leq w_{J}^{k}, \quad J \subseteq J^{d}, k \in N \backslash E,
\end{array}
$$

are also implicit in the linear description of $P_{\mathrm{RLT}}^{d}$. The argument is obvious for constraints (35). For constraints (36), let $k$ be an index belonging to $N \backslash E$ and let $J$ be a subset from $J^{d}$, and the following constraint

$$
x_{k}+\prod_{j \in J} x_{j}\left(1-x_{k}\right) \leq 1
$$

is valid for $\widehat{P}_{\mathrm{RLT}}^{d}$. Using RLT linearization, we get the constraint

$$
x_{k}+w_{J}-w_{J}^{k} \leq 1
$$

Combining this last constraint with constraint (34) we deduce that

$$
x_{k}+\sum_{j \in J} x_{j}-|J| \leq w_{J}^{k}, \quad J \subseteq J^{d}, k \in N \backslash E .
$$

Now consider the following identifications:

$$
\begin{aligned}
& w_{J}=t_{J} \quad \forall J \subseteq E,|J| \leq \min (d+1, n), \\
& w_{J}^{k}=t_{J}^{k}
\end{aligned}
$$

for every $k \in N \backslash E, J \subseteq E,|J| \leq \min (d, n)$.

These identifications imply identifications between the $W$ and $T$ variables through (12) and (32). Thus, the two extended linear descriptions $P_{\mathrm{RLT}}^{d}$ and $P_{\mathrm{RTC}}^{d}$ are equivalent. This completes the proof.

Consequently, the rank- $n$ RTC relaxation coincides with the convex hull of the mixed integer set $X$. The RTC hierarchy is motivated by the next theorem where it is shown that the projection onto the $x$-space of any RTC relaxation can be sandwiched between two convex sets. The following proposition will be useful.

Proposition 5. Let $J$ and $H$ be two disjoint subsets from $N$, such that $x_{j}$ belongs to $[0,1]$ for all $j$ belonging to $J \cup H$; then

$$
\begin{gathered}
\left(\sum_{j \in J} x_{j}+\sum_{j \in H}\left(1-x_{j}\right)-|J \cup H|+1\right)^{+} \\
\leq \min \left\{\min _{h \in J}\left\{x_{h}\right\}, \min _{h \in H}\left\{1-x_{h}\right\}\right\} .
\end{gathered}
$$

Theorem 6. For every integerd belonging to $\{1, \ldots, n\}$, one has

$$
\operatorname{conv}\left(\mathscr{T}^{d} \cap \mathscr{E}_{t}^{d}\right) \subseteq \widehat{P}_{\mathrm{RTC}}^{d} \subseteq \mathscr{T}^{d} .
$$

Proof. First, to prove the left inclusion in (42) it is sufficient to prove that the set $\left(\mathscr{T}^{d} \cap \mathscr{E}_{t}^{d}\right)$ is included in $\widehat{P}_{\mathrm{RTC}}^{d}$. Let $\widehat{x}$ be a point belonging to the set $\mathscr{T}^{d} \cap \mathscr{E}_{t}^{d}$. Let $(\widehat{x}, \widehat{t}, \widehat{v})$ be a vector such that

$$
\begin{aligned}
& \widehat{t}_{J}=\min \left\{\widehat{x}_{j}: j \in J\right\}, \quad J \subset E,|J| \leq \min \{d+1, n\}, \\
& \widehat{t}_{J}^{k}=\min \left\{\widehat{x}_{j}: j \in J \cup\{k\}\right\}, \\
& k \in N \backslash E, J \subset J^{d}, J^{d} \in E^{[d]}, \\
& \widehat{v}_{J}=\left(\sum_{j \in J} \widehat{x}_{j}-|J|+1\right)^{+}, \\
& \widehat{v}_{J}^{k}=\left(\widehat{x}_{k}+\sum_{j \in J} \widehat{x}_{j}-|J|+2\right)^{+}, \\
& k \in N \backslash E, J \subset J^{d}, J^{d} \in E^{[d]} .
\end{aligned}
$$

Thus, by definition constraints (31) are fulfilled by the vector $(\widehat{x}, \widehat{t}, \widehat{v})$. Since $\widehat{x}$ belongs to $\mathscr{T}^{d}$, then $(\widehat{x}, \widehat{t}, \widehat{v})$ satisfies constraints $(23)-(26)$. By Proposition 5 the point $(\hat{x}, \widehat{t}, \widehat{v})$ satisfies constraints (27)-(30). Since the vector $\widehat{x}$ also belongs to the 
set $\mathscr{E}_{t}^{d}$, then the vector $(\widehat{x}, \widehat{t}, \widehat{v})$ also satisfies constraints (21). Consequently, the vector $(\widehat{x}, \hat{t}, \widehat{v})$ belongs to $P_{\mathrm{RTC}}^{d}$. Thus, $\widehat{x}$ belongs to $\widehat{P}_{\mathrm{RTC}}^{d}$.

Now, let us prove the right inclusion in (42). Let $\widehat{x}$ be a point belonging to $\widehat{P}_{\mathrm{RTC}}^{d}$. There is a vector $\hat{t}$ such that $(\widehat{x}, \hat{t})$ belongs to $P_{\mathrm{RTC}}^{d}$. Without loss of generality, any constraint defining $P_{\mathrm{RTC}}^{d}$ can be written as follows:

$$
\begin{aligned}
& \sum_{J \in S_{1}^{+}} a_{J}^{+} t_{J}+\sum_{J \in S_{2}^{+}, k \in N \backslash E} b_{J}^{+} t_{J}^{k}+\sum_{J \in S_{1}^{-}} a_{J}^{-} t_{J}+\sum_{J \in S_{2}^{-}, k \in N \backslash E} b_{J}^{-} t_{J}^{k} \\
& \quad \leq 0
\end{aligned}
$$

where $S_{1}^{+}, S_{2}^{+}, S_{1}^{-}$, and $S_{2}^{-}$are subsets of the power set of $N$ (the superscript of a coefficient indicates its sign). Since the point $(\widehat{x}, \widehat{t})$ satisfies the following inequalities:

$$
\begin{array}{r}
\left(\sum_{j \in J} \widehat{x}_{j}-|J|+1\right)^{+} \leq \widehat{t}_{J} \leq \min \left\{\widehat{x}_{j}: j \in J\right\}, \\
J \subseteq J^{d}, \\
\left(\widehat{x}_{k}+\sum_{j \in J} \widehat{x}_{j}-|J|\right)^{+} \leq \widehat{t}_{J}^{k} \leq \min \left\{\widehat{x}_{j}: j \in J \cup\{k\}\right\}, \\
J \subseteq J^{d}, k \in N \backslash E,
\end{array}
$$

then we deduce

$$
\begin{aligned}
\sum_{J \in S_{1}^{+}} a_{J}^{+} & \left(\sum_{j \in J} \widehat{x}_{j}-|J|+1\right)^{+} \\
& +\sum_{J \in S_{2}^{+}, k \in N \backslash E} b_{J}^{+}\left(\widehat{x}_{k}+\sum_{j \in J} \widehat{x}_{j}-|J|\right)^{+} \\
& +\sum_{J \in S_{1}^{-}} a_{J}^{-} \min _{j \in J}\left\{\widehat{x}_{j}\right\}+\sum_{J \in S_{2}, k \in N \backslash E} b_{J}^{-} \min _{j \in J \cup\{k\}}\left\{\widehat{x}_{j}\right\}
\end{aligned}
$$

$\leq 0$.

Consequently, the point $\hat{x}$ satisfies all constraints defining $\mathscr{T}^{d}$ : that is, $\widehat{P}_{\text {RTC }}^{d} \subseteq \mathscr{T}^{d}$. This completes the proof.

Characterization (42) will allow us, in some cases (see Corollary 8), to give an explicit characterization of any RLT relaxation (characterization in the $x$-space). Before answering this question we will recall, in the next proposition, the simple result stating that any fractional point belonging to the set $\mathscr{E}_{t}$ will belong to either a facet or an edge of the hypercube. This implies that the set $\mathscr{E}_{t}$ has empty interior.

Proposition 7. For avery subset $J$ from $N$, such that $0<x_{j} \leq$ 1 for all $j$ belonging to the set $J$, if

$$
\left(\sum_{j \in J} x_{j}-|J|+1\right)^{+}=\min \left\{x_{j}: j \in J\right\},
$$

then at least $|J|-1$ variables are equal to 1.

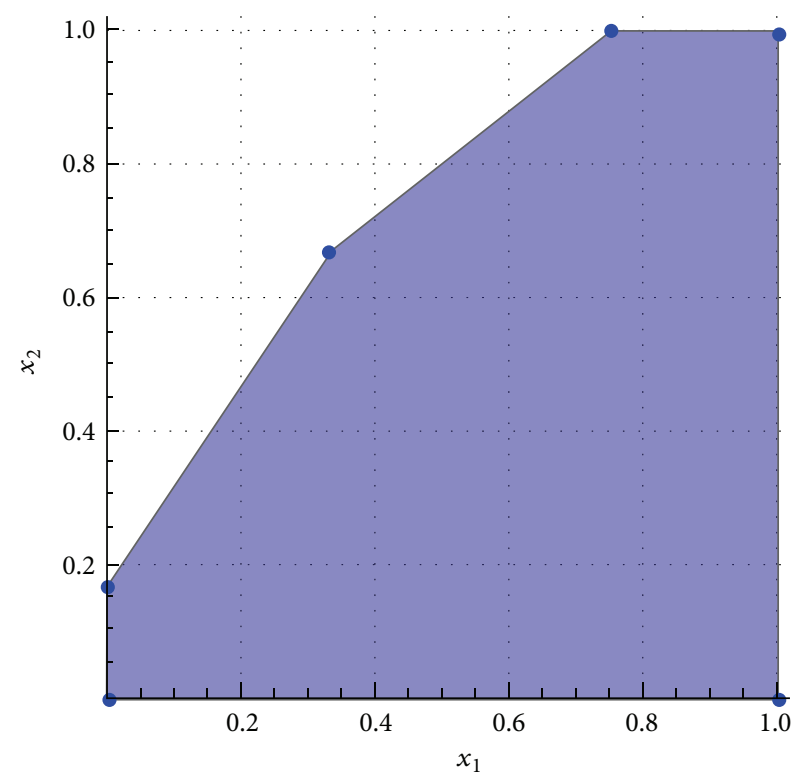

Figure 1: The set $\bar{X}$.

As a consequence of Theorem 6 and Proposition 7 we have the following corollary.

Corollary 8. For every integer $d$ belonging to $\{1, \ldots, n\}$, if the set $\mathscr{T}^{d}$ has integer vertices then it coincides with the projection of $P_{\mathrm{RLT}}^{d}$ onto the $x$-space.

Proof. Let $d$ be an integer belonging to $\{1, \ldots, n\}$. On the one hand, the sets $\mathscr{T}^{d}$ and $\mathscr{E}_{t}^{d}$ are both subsets from the hypercube. On the other hand, if the set $\mathscr{T}^{d}$ has integer vertices then both sets $\mathscr{T}^{d} \cap \mathscr{E}_{t}^{d}$ and $\mathscr{T}^{d}$ have the same vertices (vertices of $\mathscr{T}^{d}$ ). Thus, the convex envelope of the set $\mathscr{T}^{d} \cap \mathscr{E}_{t}^{d}$ coincides with the set $\mathscr{T}^{d}$, because $\mathscr{T}^{d}$ is convex. Consequently, using Theorems 4 and 6 we conclude that

$$
\widehat{P}_{\mathrm{RLT}}^{d}=\mathscr{T}^{d} .
$$

This completes the proof.

The following example shows that restricting the set $\mathscr{T}^{d}$ to have integer vertices in Corollary 8 is not a sufficient condition to characterize $\widehat{P}_{\mathrm{RLT}}^{d}$. Let us consider the following set:

$$
\begin{aligned}
X & =\left\{(x, y) \in\{0,1\}^{2}:-9 x+6 y \leq 1,-12 x+15 y\right. \\
& \leq 6\} .
\end{aligned}
$$

Its continuous relaxation $\bar{X}$ is the shaded region drawn in Figure 1. The set $\mathscr{T}^{1}$ associated with the set $X$ is the shaded region drawn in Figure 2. A careful analysis of the set $\mathscr{T}^{1}$ shows that it features the following linear description:

$$
\begin{aligned}
& \mathscr{T}^{1} \\
& \quad=\left\{(x, y) \in[0,1]^{2}:-5 x+6 y \leq 1,-4 x+3 y \leq 0\right\} .
\end{aligned}
$$




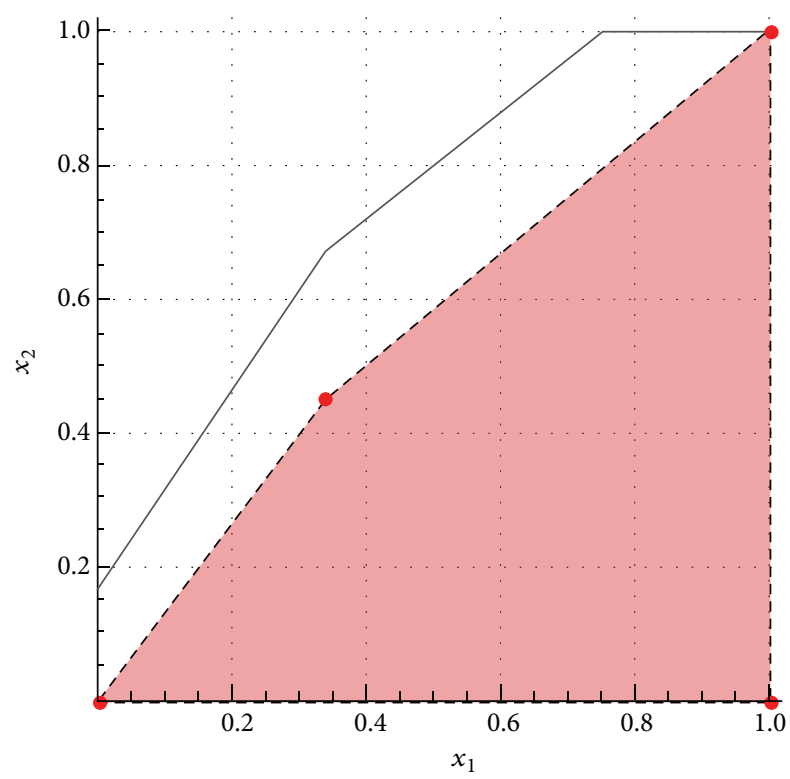

Figure 2: The set $\mathscr{T}^{1}$.

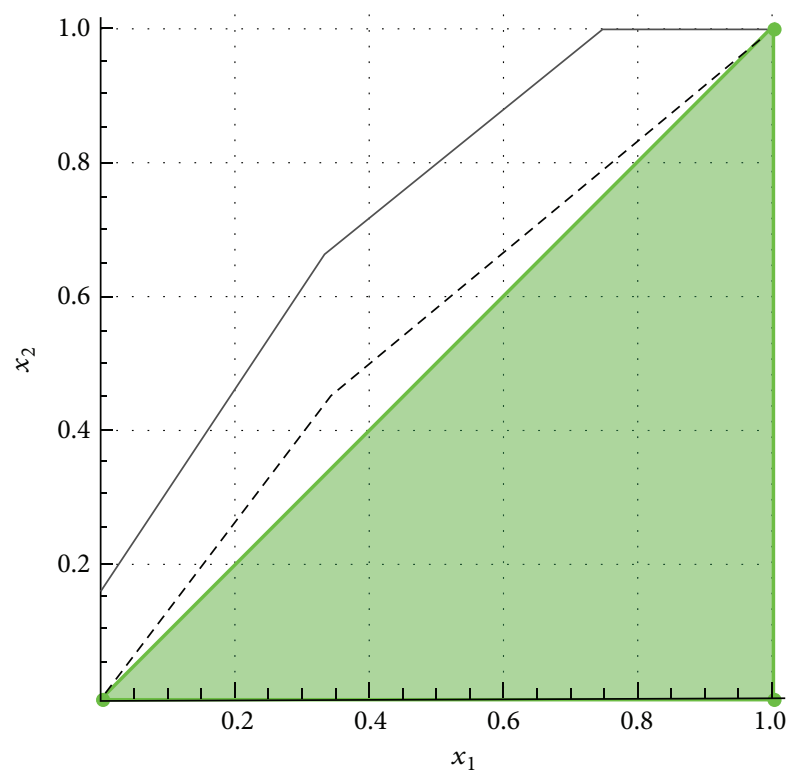

FIgURE 3: The set $\mathscr{T}^{2}$.

The rank-1 RLT relaxation has the same linear description as $\mathscr{T}^{1}$. But, as shown in Figure 2, the set $\mathscr{T}^{1}$ has a fractional vertex. Notice that set (49) coincides with the the rank-1 L\&P relaxation. As discussed in Section 5, this equality is not true in general. Finally, the set $\mathscr{T}^{2}$ (the shaded region in Figure 3) has integer vertices and it coincides with the rank-2 RLT relaxation.

3.2. Reformulation-Standard-Convexification Hierarchy. Contrary to the RTC hierarchy, in the reformulationstandard-convexification hierarchy (RSC) we convexify each factor obtained after reformulation and not the monomials appearing in each such factors. The term standard extension was introduced by Crama (see [24]) in studying concave envelopes of pseudo-boolean functions.

Let $\mathcal{S}^{d}$ be the nonlinear convex set:

$$
\mathcal{S}^{d}=\bigcap_{J^{d} \in E^{[d]}} \mathcal{S}^{d}\left(J^{d}\right),
$$

where, for each cardinality $d$ subset $J^{d}$ of $E$, the convex set $\mathcal{S}^{d}\left(J^{d}\right)$ corresponds to the solution set of $R^{d}\left(J^{d}\right)$ defined by the nonlinear system deduced from (7) using the following convexification scheme:

$$
\begin{aligned}
& \mathscr{L}\left(\alpha \prod_{k \in H} x_{k} \prod_{j \in K}\left(1-x_{j}\right)\right) \\
& = \begin{cases}\alpha\left(\sum_{j \in H} x_{j}+\sum_{j \in K}\left(1-x_{j}\right)-|K \cup H|+1\right)^{+} & \text {if } \alpha>0, \\
\alpha \min \left\{\min _{j \in H}\left\{x_{j}\right\}, \min _{k \in \mathrm{K}}\left\{1-x_{k}\right\}\right\} & \text { if } \alpha \leq 0,\end{cases}
\end{aligned}
$$

where $H$ and $K$ are two subsets such that $K \subset E, H \subset N$, and $|H \cap(N \backslash E)| \leq 1$.

As for the $l$ operator, depending on the type of the constraint $(\geq$ or $\leq)$, we use the min expression or the max expression in such a way that the resulting solution set will be convex.

The nonlinear description of the set $\mathcal{S}^{d}\left(J^{d}\right)$, for a given $d$ and a set $J^{d}$, is thus defined as follows:

$$
\begin{aligned}
& \sum_{j \in N} \mathscr{L}_{j}^{J, J^{d}}\left[a_{i j}\right]+\mathscr{L}_{0}^{J, J^{d}}\left[-b_{i}\right] \leq 0, \\
& J \subseteq J^{d}, i=1, \ldots, \rho, \\
& \mathscr{L}_{j}^{J J^{d}}[1]+\mathscr{L}_{0}^{J J^{d}}[-1] \leq 0, \quad j \in E, J \subseteq J^{d}, \\
& \mathscr{L}_{j}^{J J^{d}}[-1] \leq 0, \quad j \in N, J \subseteq J^{d}, \\
& \mathscr{L}_{0}^{J J^{d}}[-1] \leq 0, \quad J \subseteq J^{d},
\end{aligned}
$$

where, for every index $j$ belonging to $N$ and any scalar $\sigma$, $\mathscr{L}_{j}^{J, J^{d}}[\sigma]$ and $\mathscr{L}_{0}^{J, J^{d}}[\sigma]$ denote the convexified forms of the polynomials $\sigma x_{j} F_{d}\left(J, J^{d} \backslash J\right)$ and $\sigma F_{d}\left(J, J^{d} \backslash J\right)$ using the operator $\mathscr{L}$, respectively.

Since the set $\mathcal{S}^{d}$ as defined in (51) is the intersection of convex sets then we have the following results.

Theorem 9. For every integer $d$ belonging to $\{1, \ldots, n\}$, the set $\mathcal{S}^{d}$ as defined by (51) is a nonlinear convex relaxation of the mixed integer set $X$.

For every integer $d$, let $\mathscr{E}_{s}^{d}$ be the set (the lower-script $s$ is used to recall that our set is related to the standard convexification scheme)

$$
\mathscr{E}_{s}^{d}=\bigcap_{J^{d} \in E^{[d]},}{ }_{K \in\left(N \backslash J^{d}\right)^{(1)}} \mathscr{E}_{s}\left(J^{d}, K\right),
$$


where for subsets $J^{d} \in E^{[d]}$ and $K \in\left(N \backslash J^{d}\right)^{(1)}$ the set $\mathscr{C}_{s}\left(J^{d}, K\right)$ is the subset from $[0,1]^{n+m}$ defined by the following constraints:

$$
\begin{aligned}
& \left(\sum_{j \in J \cup K} x_{j}+\sum_{j \in J^{d} \backslash J}\left(1-x_{j}\right)-d+1\right)^{+} \\
& =\min \left\{\min \left\{x_{j}: j \in J \cup K\right\},\right. \\
& \left.\quad \min \left\{1-x_{j}: j \in J^{d} \backslash J\right\}\right\}, \quad J \subseteq J^{d} .
\end{aligned}
$$

Since every binary vector from $\{0,1\}^{n+m}$ belongs to $\mathscr{E}_{s}^{d}$ then the set $\operatorname{conv}\left(\mathscr{E}_{s}^{d}\right)$ coincides with the hypercube $[0,1]^{n+m}$. Notice that the set $\mathscr{E}_{s}^{d}$ is a subset from $\mathscr{E}_{t}^{d}$. As shown below, it is possible to represent $\mathcal{S}^{d} \cap \mathscr{E}_{s}^{d}$ as a polyhedron in some appropriate extended space.

Let $P_{\mathrm{RSC}}^{d}$ denote the extended linear description of the set $\mathcal{S}^{d} \cap \mathscr{E}_{s}^{d}$ obtained by using the following steps. First, let $G$ and $D$ be two sets of variables such that, for every $d$-element set $J^{d}$, for every subset $J$ from $J^{d}$, and for every subset $K$ from $N \backslash J^{d}$ with at most one element, $G_{K}^{J J^{d}}$ replaces in (53) the nonlinear term:

$$
\min \left\{\min _{h \in J \cup K}\left\{x_{h}\right\}, \min _{k \in J^{d} \backslash J}\left\{1-x_{k}\right\}\right\},
$$

and the variable $D_{K}^{J J^{d}}$ replaces

$$
\left(\sum_{h \in J \cup K} x_{h}+\sum_{k \in J^{d} \backslash J}\left(1-x_{k}\right)-d+1\right)^{+} .
$$

Then we impose the following equality:

$$
G_{K}^{J J^{d}}=D_{K}^{J J^{d}}
$$

In the sequel, instead of $G_{K}^{J J^{d}}$ or $D_{K}^{J J^{d}}$ we will use $G_{j}^{J J^{d}}$ or $D_{j}^{J J^{d}}$ when the set $K$ coincides with the singleton $\{j\}$ and $G_{0}^{J, J^{d}}$ or $D_{0}^{J, J^{d}}$ otherwise (recall that the set $K$ has at most one element).

Thus, we obtain the extended linear description:

$$
P_{\mathrm{RSC}}^{d}=\bigcap_{J^{d} \in E^{[d]}} P_{\mathrm{RSC}}^{d}\left(J^{d}\right)
$$

where, for each subset $J^{d}$ of $E$, the polyhedron $P_{\mathrm{RSC}}^{d}\left(J^{d}\right)$ reads

$$
\begin{aligned}
& \sum_{j \in N} a^{j} G_{j}^{J J J^{d}}-b G_{0}^{J J^{d}} \leq 0, \quad J \subseteq J^{d}, \\
& G_{j}^{J J^{d}}-G_{0}^{J J^{d}} \leq 0, \\
& j \in E, J \subseteq J^{d}, \\
& -G_{j}^{J J^{d}} \leq 0, \\
& j \in N, J \subseteq J^{d}, \\
& -G_{0}^{J J^{d}} \leq 0, \quad J \subseteq J^{d}, \\
& \min \left\{x_{k}: k \in J \cup\{j\}\right\} \geq G_{j}^{J J^{d}}, \\
& j \in N \backslash J^{d}, J \subseteq J^{d}, \\
& \min \left\{1-x_{k}: k \in J^{d} \backslash J\right\} \geq G_{j}^{J J^{d}}, \\
& j \in N \backslash J^{d}, J \subseteq J^{d}, \\
& \min \left\{x_{k}: k \in J\right\} \geq G_{0}^{J J^{d}}, \quad J \subseteq J^{d}, \\
& \min \left\{1-x_{k}: k \in J^{d} \backslash J\right\} \geq G_{0}^{J J^{d}}, \quad J \subseteq J^{d}, \\
& \sum_{h \in J \cup\{j\}} x_{h}+\sum_{k \in J^{d} \backslash J}\left(1-x_{k}\right)-d+1 \leq G_{j}^{J J^{d}}, \\
& j \in N \backslash J^{d}, J \subseteq J^{d}, \\
& \sum_{h \in J} x_{h}+\sum_{k \in J^{d} \backslash J}\left(1-x_{k}\right)-d+1 \leq G_{0}^{J J^{d}}, \quad J \subseteq J^{d} .
\end{aligned}
$$

Notice that linear description (60)-(69) are stated using only the variables $G$. This is possible according to (58). As discussed in the last section, discarding constraints (58) in the definition of the RSC hierarchy will lead to a weaker hierarchy.

For every integer $d$ belonging to $\{1, \ldots, n\}$, let $\widehat{P}_{\mathrm{RSC}}^{d}$ be the projection onto the $x$-space of the extended linear description $P_{\mathrm{RSC}}^{d}$. The continuous relaxation $\widehat{P}_{\mathrm{RSC}}^{\mathrm{d}}$ will be called rank$d$ reformulation-standard-convexification relaxation of the mixed integer set $X$.

As for the RTC hierarchy, in the following theorem we will prove that any relaxation of the RSC hierarchy can also be sandwiched between two convex sets.

Theorem 10. For every integer d belonging to $\{1, \ldots, n\}$, we have

$$
\operatorname{conv}\left(\mathcal{S}^{d} \cap \mathscr{E}_{s}^{d}\right) \subseteq \widehat{P}_{\mathrm{RSC}}^{d} \subseteq \mathcal{S}^{d} .
$$

Proof. Let $d$ be an integer belonging to $\{1, \ldots, n\}$. First, to prove the left inclusion in (70) it is sufficient to prove that the set $\mathcal{S}^{d} \cap \mathscr{E}_{s}^{d}$ is a subset of $\widehat{P}_{\mathrm{RSC}}^{d}$. Let $\widehat{x}$ be a point belonging to the set $\mathcal{S}^{d} \cap \mathscr{E}_{s}^{d}$. Let $(\widehat{x}, \widehat{G}, \widehat{D})$ be a vector where the two vectors $\widehat{G}$ and $\widehat{D}$ are defined as follows: for every $d$-element 
set $J^{d}$, for every subset $J$ from $J^{d}$, and for every subset $K$ from $N \backslash J^{d}$ with at most one element:

$$
\begin{aligned}
& \widehat{G}_{K}^{I J^{d}}=\min \left\{\min _{h \in J \cup K}\left\{\widehat{x}_{h}\right\}, \min _{k \in J^{d} \backslash J}\left\{1-\widehat{x}_{k}\right\}\right\}, \\
& \widehat{D}_{K}^{I J^{d}}=\left(\sum_{h \in J \cup K} \widehat{x}_{h}+\sum_{k \in J^{d} \backslash J}\left(1-\widehat{x}_{k}\right)-d+1\right)^{+} .
\end{aligned}
$$

By definition, $\widehat{x}$ belongs to $\mathcal{S}^{d}$; then $(\widehat{x}, \widehat{G}, \widehat{D})$ satisfies constraints $(60)-(63)$. By Proposition 5 the point $(\widehat{x}, \widehat{G}, \widehat{D})$ also satisfies constraints (64)-(69). Since the vector $\widehat{x}$ also belongs to the set $\mathscr{E}_{s}^{d}$, then the vector $(\widehat{x}, \widehat{G}, \widehat{D})$ also satisfies constraints (58). Thus, the vector $(\widehat{x}, \widehat{G}, \widehat{D})$ belongs to $P_{\mathrm{RSC}}^{d}$. Consequently, $\widehat{x}$ belongs to $\widehat{P}_{\mathrm{RSC}}^{d}$. This completes the first part of the proof.

Now, let us show the right inclusion in (70). Let $\hat{x}$ be a point belonging to $\widehat{P}_{\mathrm{RSC}}^{d}$. There is a vector $\widehat{G}$ such that $(\widehat{x}, \widehat{G})$ belongs to $P_{\mathrm{RSC}}^{d}$. Without loss of generality, each constraint in (60) and (61) can be rewritten as follows:

$$
\sum_{j \in N: \alpha_{i j}>0} \alpha_{i j} G_{j}^{J j^{d}}+\sum_{j \in N: \alpha_{i j}<0} \alpha_{i j} G_{j}^{J J^{d}}-\beta_{i} G_{0}^{J J^{d}} \leq 0,
$$

$$
J \subseteq J^{d} .
$$

Without loss of generality, we can assume that $\beta_{i}$ is nonnegative (the argument we will use holds also in the case where $\beta_{i}$ is nonpositive). The point $(\widehat{x}, \widehat{G})$ satisfies also the constraints:

$$
\begin{aligned}
& \min \left\{\widehat{x}_{k}: k \in J\right\} \geq \widehat{G}_{0}^{J J^{d}}, \\
& J \subseteq J^{d}, \\
& \min \left\{1-\widehat{x}_{k}: k \in J^{d} \backslash J\right\} \geq \widehat{G}_{0}^{J, J^{d}}, \\
& J \subseteq J^{d} \\
& \left(\sum_{h \in J} \widehat{x}_{h}+\sum_{k \in J^{d} \backslash J}\left(1-\widehat{x}_{k}\right)-d+1\right)^{+} \leq \widehat{G}_{0}^{J, J^{d}}, \\
& J \subseteq J^{d}, \\
& \min \left\{\widehat{x}_{k}: k \in J \cup\{j\}\right\} \geq \widehat{G}_{j}^{J J^{d}}, \\
& j \in N \backslash J^{d}, J \subseteq J^{d}, \\
& \min \left\{1-\widehat{x}_{k}: k \in J^{d} \backslash J\right\} \geq \widehat{G}_{j}^{J, J^{d}}, \\
& j \in N \backslash J^{d}, J \subseteq J^{d}, \\
& \left(\sum_{h \in J \cup\{j\}} \widehat{x}_{h}+\sum_{k \in J^{d} \backslash J}\left(1-\widehat{x}_{k}\right)-d+1\right)^{+} \leq \widehat{G}_{j}^{J J^{d}}, \\
& j \in N \backslash J^{d}, J \subseteq J^{d} .
\end{aligned}
$$

Thus, we have

$$
\begin{aligned}
& \sum_{j \in N: \alpha_{i j}>0} \alpha_{i j}\left(\sum_{h \in J} \widehat{x}_{h}+\sum_{k \in J^{d} \backslash J}\left(1-\widehat{x}_{k}\right)-d+1\right)^{+} \\
& \quad+\sum_{j \in N: \alpha_{i j}<0} \alpha_{i j} \min \left\{\min _{k \in J\{j\}\}}\left\{\widehat{x}_{k}\right\}, \min _{k \in J^{d} \backslash J}\left\{1-\widehat{x}_{k}\right\}\right\} \\
& \quad-\beta_{i} \min \left\{\min _{k \in J}\left\{\widehat{x}_{k}\right\}, \min _{k \in J^{d} \backslash J}\left\{1-\widehat{x}_{k}\right\}\right\} G_{0}^{J J^{d}} \\
& \leq \sum_{j \in N: \alpha_{i j}>0} \alpha_{i j} G_{j}^{J, J^{d}}+\sum_{j \in N: \alpha_{i j}<0} \alpha_{i j} G_{j}^{J, J^{d}}-\beta_{i} G_{0}^{J J^{d}}, \\
& J \subseteq J^{d} .
\end{aligned}
$$

This means that the point $\hat{x}$ satisfies constraints (53). Thus, $\widehat{x}$ belongs to $\mathcal{S}^{d}$. This completes the proof.

\section{Links between RTC, RSC, and L\&P Extended Linear Descriptions}

We will focus, in this section, on the connections between the extended linear descriptions of the RTC, RSC, and L\&P relaxations. First, we will compare the strength of the RTC and RSC hierarchies. We will prove that for every rank $d$ the relaxation $\widehat{P}_{\mathrm{RTC}}^{d}$ dominates the relaxation $\widehat{P}_{\mathrm{RSC}}^{d}$.

Let $I=\left\{i_{1}, \ldots, i_{p}\right\}$ be a set of indices and let $j$ be an integer less than or equal to $|I|$. Let $I_{j}$ be the subset from $I$ defined as follows:

$$
I_{j}= \begin{cases}\emptyset, & \text { if } j=0, \\ \left\{i_{k} \in I: k \leq j\right\}, & \text { otherwise. }\end{cases}
$$

The following lemmas will be useful to prove the next theorem.

Lemma 11. Let I be $\left\{i_{1}, \ldots, i_{p}\right\}$ a subset of indices from $E$. Let $K$ be the set $\left\{i_{0}\right\} \cup I$, where $i_{0}$ does not belong to I. Let us consider the variables $y$ satisfying

$$
\begin{aligned}
y^{\emptyset, K} & =\sum_{H \subseteq K}(-1)^{|H|} x_{H}, \\
y^{\left\{i_{k}\right\}, K \backslash I_{k-1}} & =-\sum_{\left\{i_{k}\right\} \subseteq H \subseteq K \backslash I_{k-1}}(-1)^{|H|} x_{H}, \\
& k \in\{1, \ldots, p\} .
\end{aligned}
$$

Then, we have the following equality:

$$
y^{\emptyset,\left\{i_{0}\right\} \cup I}=\left(1-x_{i_{0}}\right)-\sum_{k=1}^{p} y^{\left\{i_{k}\right\}, K \backslash I_{k-1}} .
$$


Proof. Let $I$ be the set $\left\{i_{1}, \ldots, i_{p}\right\}$ and let $K$ be the set $\left\{i_{0}\right\} \cup I$, where $i_{0}$ does not belong to $I$. Let us consider the variables $y$ satisfying (76)-(77). First, notice that the set

$$
\begin{aligned}
& \left\{H \subseteq K: H \subseteq\left\{i_{0}\right\}\right\} \\
& \quad \cup\left\{\bigcup_{k=1}^{p}\left\{H \subseteq K:\left\{i_{k}\right\} \subseteq H \subseteq K \backslash I_{k-1}\right\}\right\}
\end{aligned}
$$

is a partition of the power set of $K$. Thus,

$$
\begin{aligned}
y^{\emptyset, K}= & \sum_{H \subseteq K}(-1)^{|H|} x_{H}, \\
= & \sum_{H \subseteq\left\{i_{0}\right\}}(-1)^{|H|} x_{H} \\
& +\sum_{k=1}^{p}\left(\sum_{\left\{i_{k}\right\} \subseteq H \subseteq K \backslash I_{k-1}}(-1)^{|H|} x_{H}\right) .
\end{aligned}
$$

Using (77) we obtain

$$
\begin{aligned}
y^{\emptyset, K} & =\sum_{H \subseteq\left\{i_{0}\right\}}(-1)^{|H|} x_{H}-\sum_{k=1}^{p} y^{\left\{i_{k}\right\}, K \backslash I_{k-1}} \\
& =1-x_{i_{0}}-\sum_{k=1}^{p} y^{\left\{i_{k}\right\}, K \backslash I_{k-1}} .
\end{aligned}
$$

Lemma 12. Let $d$ be an integer belonging to $\{1, \ldots, n\}$. Let $J^{d}$ be a d-element subset from E. Let us consider the nonnegative variables $y^{J J^{d}}$, where $J$ is a subset from $J^{d}$, satisfying

$$
y^{J J^{d}}=\sum_{J \subseteq H \subseteq J^{d}}(-1)^{|H \backslash J|} x_{H}, \quad J \subseteq J^{d} .
$$

If the variables $x$ satisfy the following inequalities:

$$
\begin{aligned}
& x_{H} \leq \min \left\{x_{j}: j \in H\right\}, \quad H \subseteq J^{d}, \\
& x_{H} \geq\left(\sum_{k \in H} x_{k}-|H|+1\right)^{+},
\end{aligned}
$$

then the variables $y$ satisfy

$$
\begin{aligned}
& y^{J J^{d}} \leq \min \left\{x_{j}: j \in J\right\}, \quad J \subseteq J^{d}, \\
& y^{J J^{d}} \leq \min \left\{1-x_{j}: j \in J^{d} \backslash J\right\}, \quad J \subseteq J^{d}, \\
& y^{J J^{d}} \geq\left(\sum_{k \in J} x_{k}-\sum_{k \in J^{d} \backslash J} x_{k}-|J|+1\right)^{+} .
\end{aligned}
$$

Proof. Let $d$ be an integer belonging to $\{1, \ldots, n\}$. Let $E$ be a finite set and let $J^{d}$ be a $d$-element subset from $E$. It is a wellknown fact that the linear transformation (82) is a bijection (see [10]) and its inverse is given by

$$
x_{J}=\sum_{J \subseteq H \subseteq J^{d}} y^{H, J^{d}}, \quad J \subseteq J^{d} .
$$

First, the variables $y^{J J^{d}}$ satisfy inequalities (85) because of (88) and (83) and the fact that the variables $y^{J, J^{d}}$ are all nonnegative. Then, using Lemma 11, which is legitimate because the variables $y^{J, J^{d}}$ are assumed nonnegative, we deduce the inequalities (86). Finally, notice that for every subset $J$ from $J^{d}$ we have

$$
x_{J} \leq y^{J J^{d}}+\sum_{k \in J^{d} \backslash J} x_{J \cup\{k\}} .
$$

Using inequalities (84) we deduce that

$$
y^{J, J^{d}}+\sum_{k \in J^{d} \backslash J} x_{J \cup\{k\}} \geq \sum_{k \in J} x_{k}-|J|+1,
$$

which is equivalent to

$$
y^{J, J^{d}} \geq \sum_{j \in J} x_{j}-\sum_{k \in J^{d} \backslash J} x_{J \cup\{k\}}-|J|+1 .
$$

Thus, for sets $J$ and $J^{d}$ the variable $y^{J J^{d}}$ satisfies inequalities (87) and this completes the proof.

Theorem 13. For every integer $d$ belonging to $\{1, \ldots, n\}$ we have

$$
\widehat{P}_{\mathrm{RTC}}^{d} \subseteq \widehat{P}_{\mathrm{RSC}}^{d}
$$

Proof. Let $d$ be an integer belonging to $\{1, \ldots, n\}$. We will prove that the extended linear description $P_{\mathrm{RTC}}^{d}$ is contained in $P_{\mathrm{RSC}}^{d}$. Let $(\hat{x}, \hat{t})$ be a point belonging to $P_{\mathrm{RTC}}^{d}$. Let us define the point $(\widehat{x}, \widehat{G})$ as follows:

$$
\begin{aligned}
& \widehat{G}_{j}^{J, J^{d}}=\sum_{J \subseteq H \subseteq J^{d}}(-1)^{|H \backslash J|} \widehat{t}_{H \cup\{j\}}, \quad J^{d}, J \subseteq J^{d}, j \in E, \\
& \widehat{G}_{j}^{J, J^{d}}=\sum_{J \subseteq H \subseteq J^{d}}(-1)^{|H \backslash J|} \widehat{t}_{H}^{j}, \quad J^{d}, J \subseteq J^{d}, j \in N \backslash E, \\
& \widehat{G}_{0}^{J, J^{d}}=\sum_{J \subseteq H \subseteq J^{d}}(-1)^{|H \backslash J|} \widehat{t}_{H}, \quad J^{d}, J \subseteq J^{d} .
\end{aligned}
$$

Since the point $(\hat{x}, \widehat{t})$ satisfies constraints (23)-(25) and (32), then it also satisfies constraints (60)-(63). Particularly, the variables $\widehat{G}$ are nonnegative. Using Lemma 12, where the variables $y$ are replaced by the variables $\widehat{G}$ and the variables $x$ are replaced by the variables $\widehat{t}$, we deduce that the point $(\widehat{x}, \widehat{G})$ satisfies constraints (66), (67), and (69). That is,

$$
\begin{array}{rr}
\min \left\{\widehat{x}_{k}: k \in J\right\} \geq \widehat{G}_{0}^{J J^{d}}, & J \subseteq J^{d}, \\
\min \left\{1-\widehat{x}_{k}: k \in J^{d} \backslash J\right\} \geq \widehat{G}_{0}^{J J^{d}}, & J \subseteq J^{d}, \\
\sum_{h \in J} \widehat{x}_{h}+\sum_{k \in J^{d} \backslash J}\left(1-\widehat{x}_{k}\right)-d+1 \leq \widehat{G}_{0}^{J J^{d}}, & J \subseteq J^{d} .
\end{array}
$$


We use the same arguments to prove that the point $(\widehat{x}, \widehat{G})$ satisfies constraints (64), (65), and (68): that is,

$$
\begin{gathered}
\min \left\{\widehat{x}_{k}: k \in J \cup\{j\}\right\} \geq \widehat{G}_{j}^{J J^{d}}, \\
j \in N \backslash J^{d}, J \subseteq J^{d}, \\
\min \left\{1-\widehat{x}_{k}: k \in J^{d} \backslash J\right\} \geq \widehat{G}_{j}^{J J^{d}}, \\
j \in N \backslash J^{d}, J \subseteq J^{d}, \\
\sum_{h \in J \cup\{j\}} \widehat{x}_{h}+\sum_{k \in J^{d} \backslash J}\left(1-\widehat{x}_{k}\right)-d+1 \leq \widehat{G}_{j}^{J J^{d}}, \\
j \in N \backslash J^{d}, J \subseteq J^{d} .
\end{gathered}
$$

Thus, by identifying $\widehat{G}_{j}^{J J^{d}}$ with $T_{j}^{J, J^{d}}$ and $\widehat{G}_{0}^{J, J^{d}}$ with $T_{0}^{J, J^{d}}$ we conclude that the point $(\widehat{x}, \widehat{G})$ satisfies all constraints (60)(63). This completes the proof.

Now, we will compare the strength of the two hierarchies RSC and L\&P. As shown in [8], the L\&P hierarchy can be obtained from the semialgebraic set (6) using a suitable linearization (for more details, see [8]). Precisely, any rank$d$ L\&P relaxation is a rank- $d$ reformulation-linearization relaxation where the linearization is performed using the following substitutions: for every subset $J^{d}$ from $E$, let

$$
\begin{aligned}
& Z_{j}^{J J^{d}}=x_{j} F_{d}\left(J, J^{d} \backslash J\right) \quad \forall j \in N, J \subseteq J^{d}, \\
& Z_{0}^{J J^{d}}=F_{d}\left(J, J^{d} \backslash J\right) \quad \forall J \subseteq J^{d} .
\end{aligned}
$$

The linearized system we obtain reads

$$
\begin{aligned}
& \sum_{j=1}^{\kappa} a^{j} Z_{j}^{J J^{d}}-b Z_{0}^{J, J^{d}} \leq 0 \forall J \subseteq J^{d}, J^{d} \in E^{[d]}, \\
& Z_{j}^{J J^{d}}-Z_{0}^{J J^{d}} \leq 0 \forall j \in E, J \subseteq J^{d}, J^{d} \in E^{[d]}, \\
& Z_{j}^{J, J^{d}}, Z_{0}^{J, J^{d}} \geq 0 \quad \forall j \in N, J \subseteq J^{d}, J^{d} \in E^{[d]} .
\end{aligned}
$$

Let $P_{\mathrm{L} \& \mathrm{P}}^{d}$ be the extended linear description (97). As before, let $\widehat{P}_{\mathrm{L} \& \mathrm{P}}^{d}$ be its projection onto the $x$-space.

In the next theorem we will prove that the hierarchy L\&P dominates the hierarchy RSC.

Theorem 14. For every integer d belonging to the set $\{1, \ldots, n\}$ we have

$$
\widehat{P}_{\mathrm{L} \& \mathrm{P}}^{d} \subseteq \widehat{P}_{\mathrm{RSC}}^{d}
$$

Proof. Let $d$ be an integer belonging to the set $\{1, \ldots, n\}$. Let $\widehat{x}$ be a point belonging to $\widehat{P}_{\mathrm{L} \& \mathrm{P}}^{d}$. There exists a variable $\widehat{Z}$ such that $(\widehat{x}, \widehat{Z})$ belongs to $P_{\mathrm{L} \& \mathrm{P}}^{d}$. Notice that, for every $d$-element subset $J^{d}$ in $E$, every subset $J$ from $J^{d}$, and for every index $j$ from $N$ we have

$$
\begin{gathered}
\left(\sum_{k \in J \cup\{j\}} x_{k}-\sum_{k \in J^{d} \backslash J} x_{k}-|J|+1\right)^{+} \leq Z_{j}^{J J^{d}} \\
\leq \min \left\{\min _{h \in J \cup\{j\}}\left\{x_{h}\right\}, \min _{k \in J^{d} \backslash J}\left\{1-x_{k}\right\}\right\}, \\
\left(\sum_{k \in J} x_{k}-\sum_{k \in J^{d} \backslash J} x_{k}-|J|+1\right)^{+} \leq Z_{0}^{J, J^{d}} \\
\leq \min \left\{\min _{h \in J}\left\{x_{h}\right\}, \min _{k \in J^{d} \backslash J}\left\{1-x_{k}\right\}\right\} .
\end{gathered}
$$

Constraints (97) and both relations (99) and (100) imply that the point $(\widehat{x}, \widehat{Z})$ satisfies constraints (60)-(69). Thus, the point $(\widehat{x}, \widehat{Z})$ belongs to $P_{\mathrm{RSC}}^{d}$. Consequently, the point $\widehat{x}$ belongs to $\widehat{P}_{\text {RSC }}^{d}$. This completes the proof.

As a byproduct of Theorem 14 we obtain an indirect proof of Theorem 13. Indeed, for any rank $d$, we know from Theorem 4 that $\widehat{P}_{\mathrm{RTC}}^{d}$ is equivalent to $\widehat{P}_{\mathrm{RLT}}^{d}$. It is a well-known fact that $\widehat{P}_{\mathrm{RLT}}^{d}$ is included in $\widehat{P}_{\mathrm{L} \& \mathrm{P}}^{d}$ (see [8]). Thus, it follows using Theorem 14 that $\widehat{P}_{\mathrm{RTC}}^{d}$ is included in $\widehat{P}_{\mathrm{RSC}}^{d}$.

\section{Weak RTC and Weak RSC Hierarchies}

In this section, we will introduce a weak version of the RTC and RSC hierarchies. For both weak hierarchies the rank $d$ extended linear description is obtained by reformulation, convexification using min and max and then linearizing using two distinct sets of variables.

More precisely, the rank $d$ extended linear description of the weak-RTC hierarchy, denoted by $P_{\mathrm{WRTC}}^{d}$, is defined as $P_{\mathrm{RTC}}^{d}$ except that we discard equality constraints (21) (see Section 3.1). Thus, both sets of variables $t_{J}^{H}$ and $v_{J}^{H}$ will appear in the description of $P_{\mathrm{WRTC}}^{d}$. Similarly, the rank $d$ extended linear description of the weak-RSC hierarchy, denoted by $P_{\mathrm{WRSC}}^{d}$, is defined as $P_{\mathrm{RSC}}^{d}$ except that we discard equality constraints (58) between the two sets of variables $G_{K}^{J J^{d}}$ and $D_{K}^{J J^{d}}$ (see Section 3.2).

To emphasize some connections (small instances are sufficient to reveal these connections. We wish to emphasize that this is not a computational investigation) between the weak hierarchies we will use the computational results shown in Table 1. The values computed are the minimum value of rank 1 RTC, L\&P, RSC, WRTC, and WRSC relaxations for five instances of the multiple constraints knapsack problem. Each instance has 5 constraints and 3 variables. The instances have been generated using the $\mathrm{Chu}$ and Beasley procedure given in [28]. The constraint matrix coefficients are integers and randomly chosen in $\{0, \ldots, 1000\}$. The right-hand-side coefficient of the $i$ th constraint is set to $0.95 \sum_{j=1}^{3} a_{i j}$. The $j$ th objective function coefficient is set to $(1 / 5) \sum_{i=1}^{5} a_{i j}+500 \zeta_{j}$, 
TABLE 1: Optimal values of the different relaxations.

\begin{tabular}{|c|c|c|c|c|c|}
\hline \multirow{2}{*}{ Instance } & \multicolumn{5}{|c|}{ Optimum values } \\
\hline & $\widehat{P}_{\mathrm{RTC}}^{1}$ & $\widehat{P}_{\mathrm{L} \& \mathrm{P}}^{1}$ & $\widehat{P}_{\mathrm{RSC}}^{1}$ & $\widehat{P}_{\mathrm{WRTC}}^{1}$ & $\widehat{P}_{\mathrm{WRSC}}^{1}$ \\
\hline inst-1 & -2676.783 & -2676.8066 & -2677.0347 & -3390.3195 & -2677.0347 \\
\hline inst-2 & -3390.3195 & -3390.7008 & -3391.2893 & -3390.3195 & -3391.2893 \\
\hline inst-3 & -3010.0385 & -3010.0385 & -3010.4338 & -3010.0385 & -3010.4338 \\
\hline inst-4 & -3229.0645 & -3229.1293 & -3229.1293 & -3229.0645 & -3229.1293 \\
\hline inst-5 & -3337.7582 & -3337.7582 & -3337.8134 & -3337.7582 & -3337.8134 \\
\hline
\end{tabular}

where $\zeta_{j}$ is a real number randomly chosen from the interval $[0,1]$.

Although RTC is stronger than RSC the WRTC and WRSC hierarchies are not comparable in strength as shown by the instances inst-1 and inst-2. The WRTC hierarchy may be stronger than L\&P hierarchy as shown by the instances inst-2 and inst-4. Also, the computational results shown in the Table 1 are coherent with the theoretical results proved before: (i) the hierarchy RTC is stronger than both L\&P and RSC hierarchies; (ii) the hierarchy L\&P is stronger than hierarchy RSC; (iii) the hierarchies RTC and RSC are stronger than WRTC and WRSC, respectively.

\section{Conclusion}

In this paper, we introduced two new hierarchies called RTC and RSC for which the rank $d$ continuous relaxations were denoted by $\widehat{P}_{\mathrm{RTC}}^{d}$ and $\widehat{P}_{\mathrm{RSC}}^{d}$, respectively. These two hierarchies are obtained using a reformulation-convexificationlinearization procedure. The hierarchy RTC is obtained using a term convexification scheme and the RSC hierarchy is obtained using a standard convexification scheme. Then we compared the strength of these two hierarchies. We proved that (i) the hierarchy RTC is equivalent to the RLT hierarchy of Sherali-Adams, (ii) the hierarchy RTC dominates the hierarchy RSC, and (iii) the hierarchy RSC is dominated by the Lift-and-Project hierarchy. Next, for every rank $d$, we proved that $\operatorname{conv}\left(\mathscr{T}^{d} \cap \mathscr{E}_{t}^{d}\right) \subseteq \widehat{P}_{\mathrm{RTC}}^{d} \subseteq \mathscr{T}^{d}$ and $\operatorname{conv}\left(\mathcal{S}^{d} \cap \mathscr{E}_{s}^{d}\right) \subseteq \widehat{P}_{\mathrm{RSC}}^{d} \subseteq \mathcal{S}^{d}$, where the sets $\mathscr{T}^{d}$ and $\mathcal{S}^{d}$ are convex, while $\mathscr{E}_{t}^{d}$ and $\mathscr{E}_{s}^{d}$ are two nonconvex sets with empty interior. The first inclusions allow, in some cases, an explicit characterization of RLT relaxations. That is a convex nonlinear description of any RLT relaxation in the $x$-space. Finally, we discussed weak version of both RTC and RSC hierarchies and emphasized some connections between them using small numerical examples.

We conclude with some open questions. First, one may ask whether the hierarchy RSC is equivalent to the hierarchy WRSC or not. Also, does the rank $n$ RSC relaxation coincide with the convex envelope of the set $X$ ? Finally, is it possible to obtain stronger hierarchies using the exposed reformulationconvexification-linearization approach? Extending this work to more general nonlinear optimization problems will be the subject of a future work.

\section{Conflict of Interests}

The author declares that there is no conflict of interests regarding the publication of this paper.

\section{Acknowledgment}

The author would like to thank the anonymous referees for their helpful comments.

\section{References}

[1] B. Korte and J. Vygen, Combinatorial Optimization: Theory and Algorithms, Algorithms and Combinatorics, Springer, 3rd edition, 2005.

[2] C. H. Papadimitriou, Computational Complexity, AddisonWesley, 1994.

[3] A. Schrijver, Combinatorial Optimization: Polyhedra and Efficiency, vol. A of Algorithms and Combinatorics, Springer, Berlin, Germany, 2003.

[4] A. Schrijver, Combinatorial Optimization: Polyhedra and Efficiency, vol. 24 of Algorithms and Combinatorics, Springer, Berlin, Germany, 2003.

[5] E. Balas, S. Ceria, and G. Cornuéjols, "A lift-and-project cutting plane algorithm for mixed 0-1 programs," Mathematical Programming, vol. 58, no. 1-3, pp. 295-324, 1993.

[6] J. B. Lasserre, "An explicit exact SDP relaxation for nonlinear 0-1 programs," in Integer Programming and Combinatorial Optimization, Lectures Notes in Computer Science, pp. 293303, Springer, Berlin, Germany, 2001.

[7] L. Lovász and A. Schrijver, "Cones of matrices and set-functions and 0-1 optimization," SIAM Journal on Optimization, vol. 1, no. 2, pp. 166-190, 1991.

[8] M. Minoux and H. Ouzia, "DRL": a hierarchy of strong blockdecomposable linear relaxations for 0-1 MIPs," Discrete Applied Mathematics, vol. 158, no. 18, pp. 2031-2048, 2010.

[9] H. D. Sherali and W. P. Adams, "A hierarchy of relaxations between the continuous and convex hull representations for zero-one programming problems," SIAM Journal on Discrete Mathematics, vol. 3, no. 3, pp. 411-430, 1990.

[10] H. D. Sherali and W. P. Adams, "A hierarchy of relaxations and convex hull characterizations for mixed-integer zero-one programming problems," Discrete Applied Mathematics, vol. 52, no. 1, pp. 83-106, 1994.

[11] H. D. Sherali and W. P. Adams, A Reformulation-Linearization Technique for Solving Discrete and Continuous Nonconvex Problems, vol. 31 of Nonconvex Optimization and Its Applications, Springer, New York, NY, USA, 1999.

[12] D. Bienstock and M. Zuckerberg, "Subset algebra lift operators for 0-1 integer programming," SIAM Journal on Optimization, vol. 15, no. 1, pp. 63-95, 2005.

[13] H. Ouzia, Hiérarchies de relaxations semi-algébriques pour des programmes linéeaires mixtes 0-1: théorie et applications [Ph.D. thesis], Université Pierre et Marie Curie, Paris, France, 2008. 
[14] M. Zuckerberg, A set theoretic approach to lifting procedures for 0-1 integer programming [Ph.D. thesis], Columbia University, New York, NY, USA, 2004.

[15] M. Laurent, "A comparison of the Sherali-Adams, LovászSchrijver, and Lasserre relaxations for 0-1 programming," Mathematics of Operations Research, vol. 28, no. 3, pp. 470-496, 2003.

[16] M. Tawarmalani and N. V. Sahinidis, Convexification and Global Optimization in Continuous and Mixed-Integer Nonlinear Programming: Theory, Algorithms, Software, and Applications, Nonconvex Optimization and its Applications, Springer-Verlag, New York, NY, USA, 2003.

[17] M. Tawarmalani and N. V. Sahinidis, "Convex extensions and envelopes of lower semi-continuous functions," Mathematical Programming, Series B, vol. 93, no. 2, pp. 247-263, 2002.

[18] J. B. Lasserre and T. P. Thanh, "Convex underestimators of polynomials," Journal of Global Optimization, vol. 56, no. 1, pp. 1-25, 2013.

[19] J. E. Falk and K. R. Hoffman, "A successive underestimation method for concave minimization problems," Mathematics of Operations Research, vol. 1, no. 3, pp. 251-259, 1976.

[20] J. P. Aubin and I. Ekeland, "Estimates of the duality gap in nonconvex optimization," Mathematics of Operations Research, vol. 1, no. 3, pp. 225-245, 1976.

[21] E. Balas and J. B. Mazzola, "Nonlinear 0-1 programming: I. Linearization techniques," Mathematical Programming, vol. 30, no. 1, pp. 1-21, 1984.

[22] H. D. Sherali and A. Alameddine, "An explicit characterization of the convex envelope of a bivariate bilinear function over special polytopes," Annals of Operations Research, vol. 25, no. 1, pp. 197-209, 1990.

[23] H. D. Sherali, "Convex envelopes of multilinear functions over a unit hypercube and over special discrete sets," Acta Mathematica Vietnamica, vol. 22, no. 1, pp. 245-270, 1997.

[24] Y. Crama, "Concave extensions for nonlinear 0-1 maximization problems," Mathematical Programming, vol. 61, no. 1, pp. 53-60, 1993.

[25] H. D. Sherali and W. P. Adams, "Reformulation-linearization techniques for discrete optimization problems," in Handbook of Combinatorial Optimization, D. Z. Du and P. M. Pardalos, Eds., pp. 479-532, Springer, New York, NY, USA, 1999.

[26] D. Cox, J. Little, and D. O'Shea, Ideals, Varieties and Algorithms, Springer, 2nd edition, 1996.

[27] D. Cox, J. Little, and D. O'Shea, Using Algebraic Geometry, Springer, 2nd edition, 2004.

[28] P. C. Chu and J. E. Beasley, "A genetic algorithm for the multidimensional knapsack problem," Journal of Heuristics, vol. 4, no. 1, pp. 63-86, 1998. 


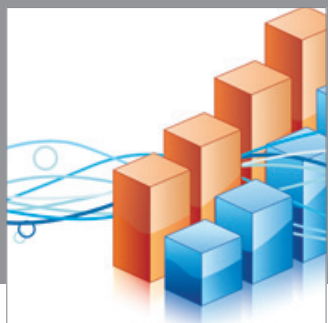

Advances in

Operations Research

mansans

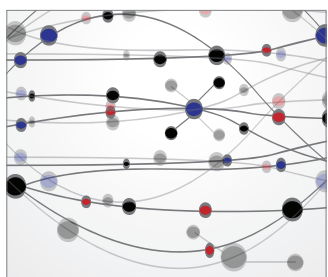

The Scientific World Journal
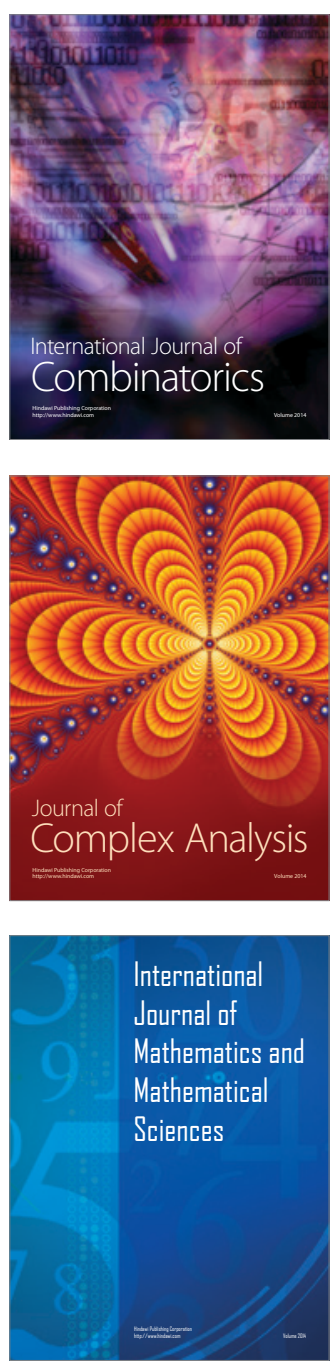
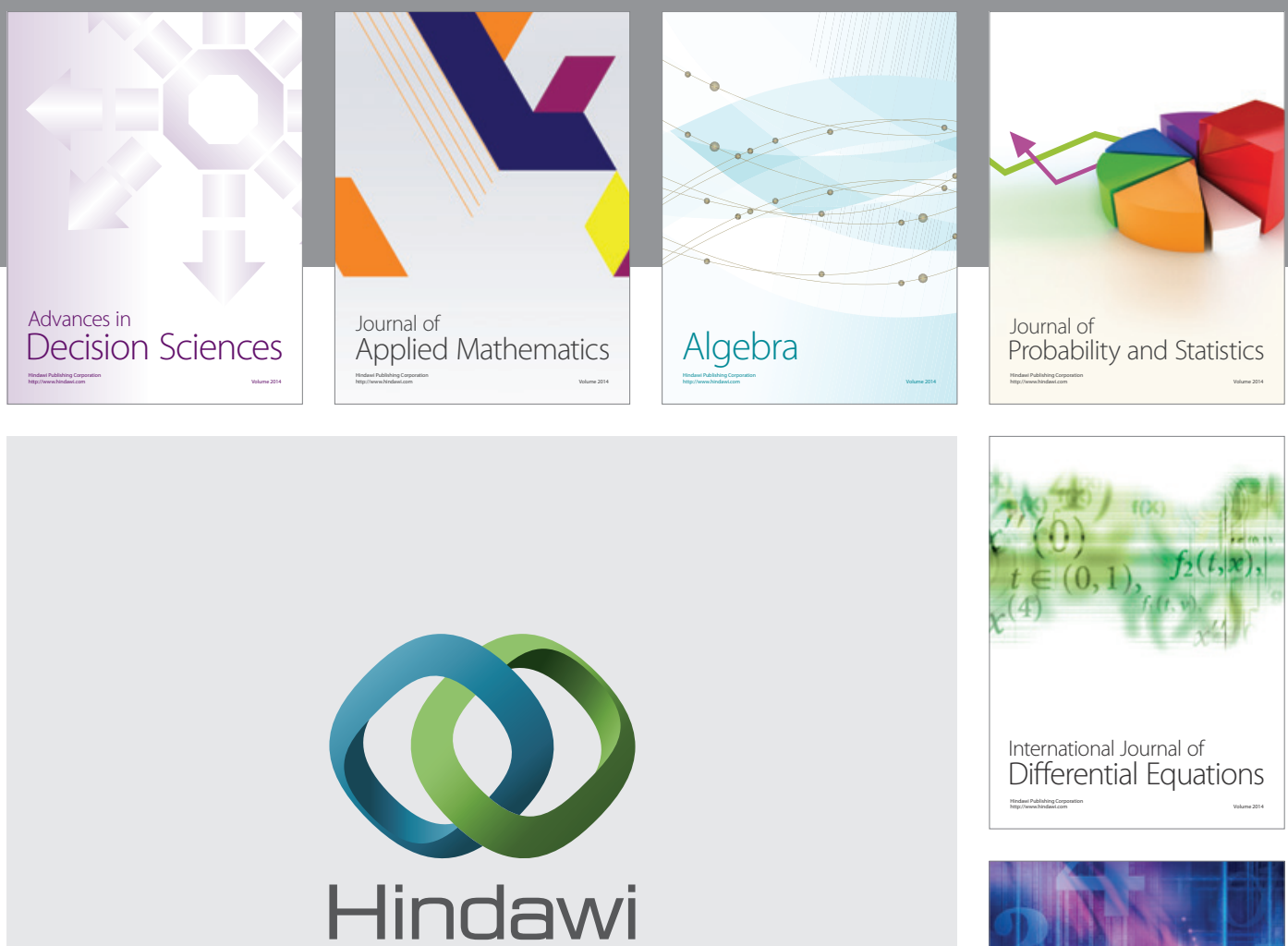

Submit your manuscripts at http://www.hindawi.com
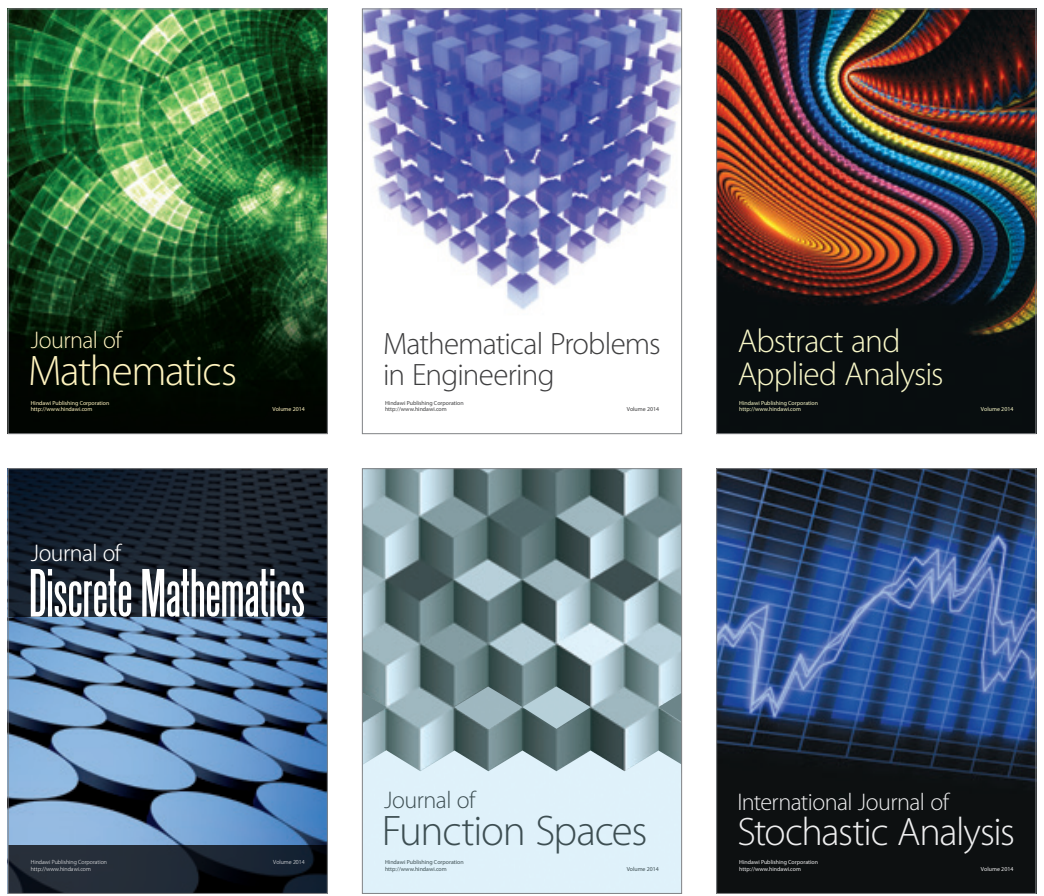

Journal of

Function Spaces

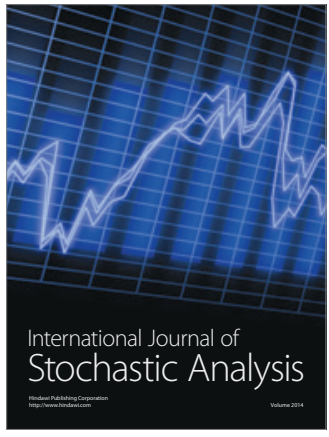

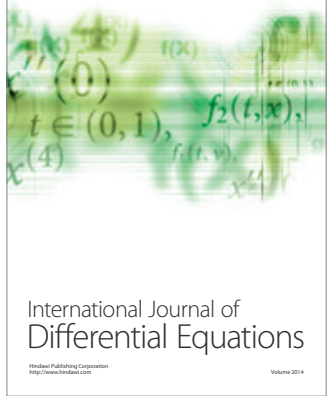
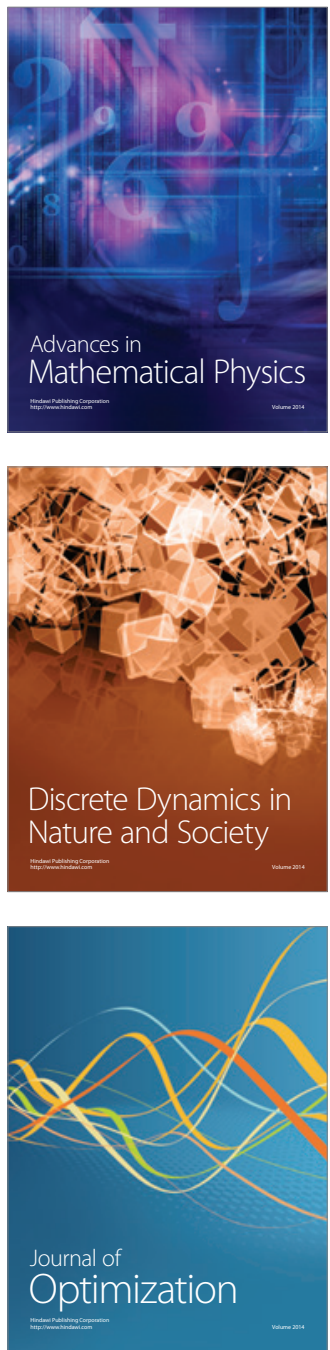